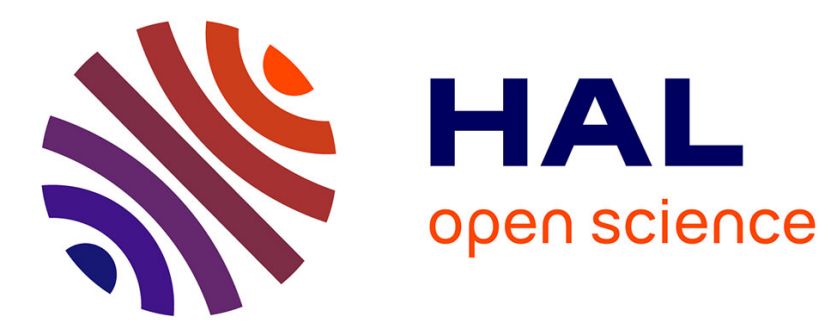

\title{
Les Maurozômai, Byzance et le sultanat de Rūm. Note sur le sceau de Jean Comnène Maurozômès
}

Sophie Métivier

\section{To cite this version:}

Sophie Métivier. Les Maurozômai, Byzance et le sultanat de Rūm. Note sur le sceau de Jean Comnène Maurozômès. Revue des études byzantines, 2009, 67 (1), pp.197-207. 10.3406/rebyz.2009.4831 . halshs-01351222

\author{
HAL Id: halshs-01351222 \\ https://shs.hal.science/halshs-01351222
}

Submitted on 4 Aug 2016

HAL is a multi-disciplinary open access archive for the deposit and dissemination of scientific research documents, whether they are published or not. The documents may come from teaching and research institutions in France or abroad, or from public or private research centers.
L'archive ouverte pluridisciplinaire HAL, est destinée au dépôt et à la diffusion de documents scientifiques de niveau recherche, publiés ou non, émanant des établissements d'enseignement et de recherche français ou étrangers, des laboratoires publics ou privés. 


\title{
LES MAUROZÔMAI, BYZANCE ET LE SULTANAT DE RŪM. NOTE SUR LE SCEAU DE JEAN COMNÈNE MAUROZÔMÈS
}

\author{
Sophie MÉTIVIER
}

Le musée de Niğde (Turquie) ${ }^{1}$ conserve le sceau de Jean Comnène Maurozômès, dont un ou plusieurs parallèles ont été signalés dans des ventes aux enchères ces dernières années ${ }^{2}$. Le sceau ${ }^{3}$ présente au droit saint Jean Prodrome, qui est nommé, de face, en pied et barbu, tenant dans sa main

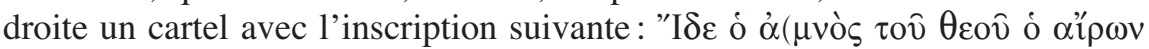

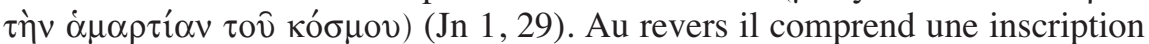

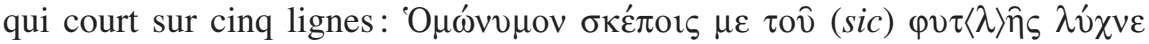

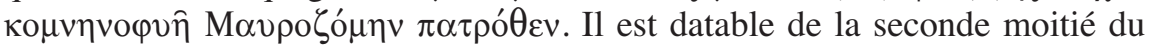
$13^{\text {e }}$ siècle. La pièce complète utilement le dossier prosopographique de la famille des Maurozômai, car elle permet d'une part d'en préciser la généalogie et l'alliance avec les Comnènes, d'autre part, puisqu'elle est conservée au musée de Niğde, d'analyser la position de cette famille, d'origine byzantine et passée au service des Seldjoukides, jusque dans la seconde moitié du $13^{\mathrm{e}}$ siècle. Elle éclaire encore, et plus généralement, les rapports entre le sultanat de Rūm et l'empire au $13^{\mathrm{e}}$ siècle.

Le propriétaire du sceau ainsi que la famille des Maurozômai, aux $12^{\mathrm{e}}$ et $13^{\mathrm{e}}$ siècles, sont connus par des sources sigillographiques et narratives ainsi que par une inscription. Les premiers personnages attestés, à savoir Jean ${ }^{4}$,

1. Je remercie M. Fazıl Açıkgöz, directeur du musée de Niğde, ainsi que Guillaume SaintGuillain et Jean-François Vannier pour leurs conseils et suggestions, André Binggeli, Étienne de la Vaissière et Éric Vallet pour leur lecture, en arabe ou en persan, des textes d'Ibn al-Athīr et d'Ibn Bībī.

2. Studies in Byzantine Sigillography [désormais abrégé en SBS] 8, p. 225 (Grün, Vente 25 [1999], no 474 : répétition de Münz Zentrum 94, n 953), p. 227 (Hirsch, Vente 196 [24-27 septembre 1997], $\mathrm{n}^{\circ}$ 1027; du même boullôtèrion, Künker 67, n 1393), p. 228 (Hirsch, Vente 199 [6-8 mai 1998], n ${ }^{\circ} 681$ : répétition de Hirsch 196, no 1027), p. 229 (Hirsch, Vente 205 [22-25 septembre 1999], n 1169: répétition de Hirsch 199, n 681), p. 239 (Münz Zentrum 94 [13-15 mai 1998], n 953), p. 251 (Schenk-Behrens, Vente 78 [7-8 octobre 1999], nº 328 : répétition de Münz Zentrum 94, $\mathrm{n}^{\circ}$ 953).

3. L'ensemble des sceaux du musée de Niğde sera publié par l'auteur dans SBS 10, 2009.

4. Sceau de l'Ermitage, daté du $12^{\mathrm{e}}$ siècle : V. A. PANČENKO, Katalog molivdovulov kollekcii Russkago Arheologičeskago Instituta v Konstantinopole, IRAIK 8, 1903, p. 230, n ${ }^{\circ} 75$; V. LAURENT, Les bulles métriques dans la sigillographie byzantine (Archives de l'Orient chrétien 2), Athènes 1932, $\mathrm{n}^{\circ} 194$; V. S̆ vulov» B. A. Pančenko, VV 38, 1977, p. 107, nº 75; SBS 5, p. 129. 
Michel ${ }^{5}$, Nicolas ${ }^{6}$ et Paul $^{7}$, le sont, exclusivement, grâce à des sceaux du $11^{\mathrm{e}} \mathrm{ou}$ du $12^{\mathrm{e}}$ siècle. Les sceaux de Jean et de Paul, similaires, présentent au droit l'effigie de la Vierge orante avec le Christ en médaillon, au revers l'inscription

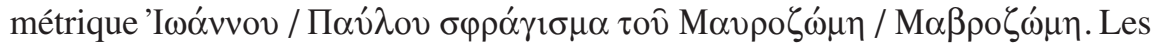
sceaux de Nicolas et de Michel ont tous deux au droit l'effigie du saint homonyme, au revers une inscription métrique dodécasyllabique 'O $\mu \omega v v \mu$ ôv $\tau \alpha$ /

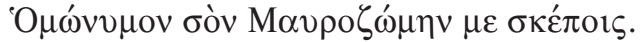

Dans la seconde moitié du $12^{\mathrm{e}}$ et dans la première moitié du $13^{\mathrm{e}}$ siècle, trois ou quatre autres Maurozômai sont connus par des sources narratives, Théodore, Jean et Manuel, ainsi qu'un émir. Le premier, officier militaire de Manuel I ${ }^{\text {er }}$, est cité deux fois par Nicétas Chôniatès, pour avoir participé, à la tête d'un détachement de la flotte, à la campagne navale conduite contre Damiette en 1168, ainsi qu'en 1176 à la bataille de Myrioképhalon, pendant laquelle il commanda l'aile gauche de l'armée byzantine ${ }^{8}$. Quoique de manière anecdotique il est encore mentionné - du moins peut-on supposer qu'il s'agit d'un seul et même individu - sous le règne d'Andronic $\mathrm{I}^{\mathrm{er}}$ par Eustathe de Thessalonique, qui le dit originaire du Péloponnèse ${ }^{9}$ et en fait un successeur de Michel Hagiothéodôritès. Suivant l'analyse de Paul Magdalino, il occuperait alors l'importante fonction de mésazôn, une fonction civile, et ce depuis le règne de Manuel Ir ${ }^{\text {er }}$. Guillaume de Tyr précise en effet que cet intime de l'empereur se vit confier la charge de tout l'empire, postérieurement à la campagne de $1169^{11}$.

Un autre Maurozômès, le sébaste Jean, est mentionné à trois reprises par Eustathe de Thessalonique dans le De expugnatione Thessalonicae. Suivant les allusions de ce dernier, Jean, un commandant militaire, gagna avec son armée, depuis le Péloponnèse, Thessalonique, pour participer à la défense de cette ville. Eustathe stigmatise sa crainte d'Andronic $\mathrm{I}^{\mathrm{er}}$ Comnène en même temps qu'il lui reproche, à mots couverts, d'être lié ou de se rallier aux Normands pour avoir conduit en Sicile, avec David Comnène, une ambassade et dans le but de prendre part au butin après la prise de la ville ${ }^{12}$.

5. Signalé dans C. Stavrakos, Die byzantinischen Bleisiegel mit Familiennamen aus der Sammlung des Numismatischen Museums Athen (Mainzer Veröffentlichungen zur Byzantinistik 4), Wiesbaden 2000, p. 260, nº 166.

6. SBS 8, p. 224 (Gorny, Vente 113 [18 octobre 2001], deuxième moitié du $12^{\mathrm{e}}$ siècle).

7. Sceau trouvé au Pirée, daté du $12^{\mathrm{e}}$ siècle. Voir K. M. Konstantopoulos, Bv $\zeta \alpha v \tau \imath \alpha \kappa \alpha \dot{\alpha}$

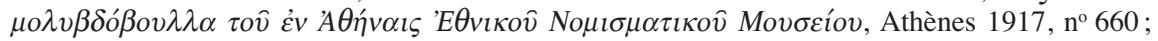
V.Laurent, Les bulles métriques, cité n. $4, \mathrm{n}^{\circ} 302$; C. Stavrakos, Die byzantinischen Bleisiegel, cité n. 5, p. 259-260, n $166 ;$ SBS 2, p. 245, no 43.

8. Nicétas Chôniatès, Histoire (CFHB 11), éd. J.-L. Van Dieten, Berlin 1975, p. 160 (Damiette), p. 180 (Myrioképhalon).

9. Eustathe de Thessalonique, De expugnatione Thessalonicae, dans Eustazio DI Tessalonica, La espugnazione di Tessalonica (Istituto siciliano di studi bizantini e neoellenici. Testi 5), éd. S. KyrIAKIDIs, Palerme 1961, p. 46.

10. P. Magdalino, The Empire of Manuel I Komnenos, 1143-1180, Cambridge 1993, p. 257258.

11. Gulllaume de Tyr, Chronique, 20, 13 (Corpus Christianorum. Continuatio Mediaevalis 63A), éd. R. B. C. Huygens, Turnhout 1986, p. 927.

12. Eustathe De Thessalonique, De expugnatione Thessalonicae, cité n. 9, p. 14, p. 88 («le sébaste Jean Maurozômès »), p. 106. Voir J.-C. CHeYnet, Pouvoir et contestations à Byzance (Byzantina Sorbonensia 9), Paris 1990, p. 432-433. 
Inédit.

Numéro d'inventaire du Musée de Niğde: 1.54.98.

Sceau cassé à gauche du champ et percé.

Diamètre : $35 \mathrm{~mm}$.

Datation: seconde moitié du $13^{\mathrm{e}}$ siècle.
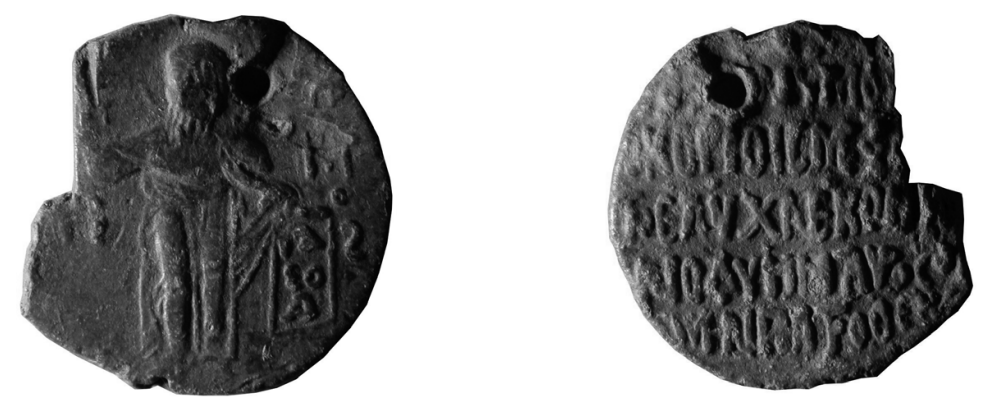

Sceau de Jean Comnène Maurozômès. Musée de Niğde (Turquie).

Au droit, saint de face, en pied, barbu, un cartel dans la main droite.

Inscription verticale à droite : $\Pi \mathrm{P}|\Delta| \mathrm{M}|\mathrm{O}| 2$

Dans le cartel : $I \triangle|\in O| A$

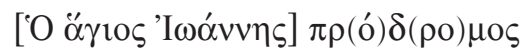

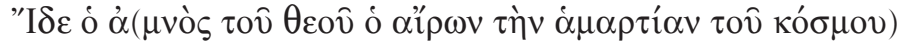

Au revers, inscription sur cinq lignes :

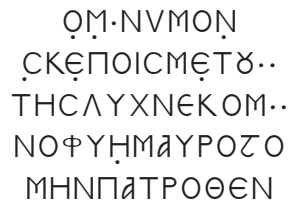

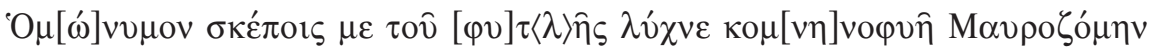
$\pi \alpha \tau \rho \theta_{\varepsilon v}$.

Je propose une autre lecture de la deuxième ligne que celle qui a été faite dans les $S B S 8$ (cité n. 2):

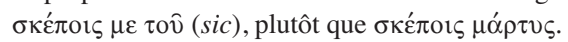


Dernier Maurozômès qui soit connu nommément (et antérieurement au sceau de Niğde), Manuel ${ }^{13}$ est évoqué exclusivement pour les relations qu'il a nouées avec le sultan de Rūm, Kaykhusraw I ${ }^{\text {er }}$, tant par Nicétas Chôniatès que dans la chronique arabe d'Ibn al-Athīr et dans la chronique seldjoukide d'Ibn $\mathrm{B} \bar{b} \mathrm{~b}$. Lorsque le sultan, évincé de Konya par son frère, se réfugie à la cour d'Alexis III, il y rencontre, entre autres dignitaires, un Maurozômès ${ }^{14}$. C'est chez ce dernier, aux environs de Constantinople, qu'il est accueilli après la prise de la Ville par les Latins (en 1203 ou en 1204?) ${ }^{15}$. Lorsqu'en 1205, ayant appris la mort du sultan Rukn al-dīn, Kaykhusraw I ${ }^{\text {er }}$ décide de regagner son trône, Maurozômès le suit en territoire seldjoukide. Entretemps le sultan (lui-même de mère chrétienne) a épousé sa fille ${ }^{16}$. Tandis que ni Nicétas Chôniatès ni Ibn Bībī ne précisent la date de ce mariage (contemporain du séjour du sultan dans l'empire), Ibn al-Athīr l'évoque avant de mentionner la prise de la Ville par les Latins et la fuite du sultan de Constantinople ${ }^{17}$. Passé au service de Kaykhusraw ${ }^{\text {er }}$, Manuel entreprend de conduire en son nom et avec des troupes turques la conquête de la vallée du Méandre. Nicétas Chôniatès affirme seul qu'il a prétendu au titre impérial en même temps que Théodore Laskaris. Bien qu'en 1206 il ait essuyé une défaite face à ce dernier ${ }^{18}$, son autorité n'en est pas moins reconnue sur ces territoires: il conserve

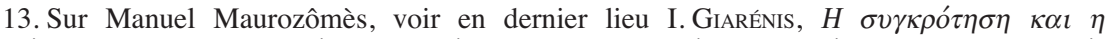

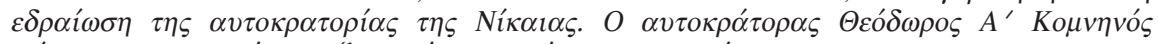

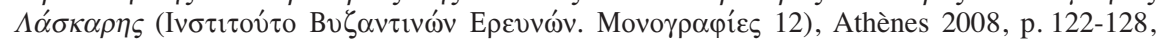
dont l'analyse de l'action politique de Manuel est modelée par les témoignages de Nicétas Chôniatès. L'auteur souligne en conséquence ses liens avec le sultan et son antagonisme avec Théodore Laskaris, sans mentionner Alexis III.

14. Iвn BīBĪ, Mukhtașar, trad. H. W. DudA, Die Seltschukengeschichte des Ibn B̄̄b̄̄, Copenhague 1959, p. 30-31. Sur la présence de Kaykhusraw I ${ }^{\text {er }}$ à la cour d'Alexis III et sur ses rapports avec l'empire, voir C. M. BRAnd, The Turkish Element in Byzantium, DOP 43, 1989, p. 12 et, surtout, D. Korobeinikov, A sultan in Constantinople: the feasts of Ghiyāth al-Dīn KayKhusraw I, dans Eat, Drink, and Be Merry (Luke 12:19) - Food and Wine in Byzantium. Papers of the 37th Annual Spring Symposium of Byzantine Studies, in Honour of Professor A. A. M. Bryer, éd. L. BrubaKer et K. Linardou, Aldershot 2007, p. 93-108.

15. IBn AL-ATHĪr, al-Kamīl fī al-tarīkh, éd. C. J. TornBerg, Louvain 1851-1871, Beyrouth 1979-1982, XII, p. 200-201 (601 H.) ; IBN BīBİ, Mukhtaṣar, cité n. 14, p. 31.

16. Cette alliance contractée dans l'empire suppose que Kaykhusraw I ${ }^{\text {er }}$ soit baptisé. C'est en effet ce qu'affirme Georges Akropolitès, Histoire, 8, éd. A. Heisenberg (Teubner), Leipzig 1903, p. 14. Sur le baptême du sultan, voir D. Korobeinikov, A sultan in Constantinople, cité n. 14, p. 101-102 (le parrain étant Alexis III).

17. IBN AL-AthĪr, al-Kamīl, cité n. 15; IBN BīBī, Mukhtașar, cité n. 14, p. 38; NicétAS

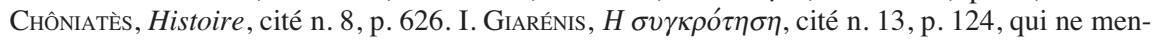
tionne que les témoignages de Nicétas Chôniatès et d'Ibn Bībī, affirme que le mariage eut lieu après que le sultan eut quitté Constantinople (en même temps qu'Alexis III) et qu'il eut rencontré Manuel Maurozômès en Asie Mineure. Aussi l'alliance de Kaykhusraw I ${ }^{\text {er }}$ avec Maurozômès est-elle analysée indépendamment de tout rapport de l'un et de l'autre avec l'empereur Alexis III.

18. NicÉTAs Chôniatès, Histoire, cité n. 8, p. 626 (avec le soutien de Kaykhusraw I ${ }^{\mathrm{er}}$, Manuel Maurozômès ravage la vallée du Méandre; Théodore Laskaris renverse Manuel, est proclamé empereur, défait David Comnène dans la région de Nicomédie, puis met en fuite Maurozômès et inflige une sévère défaite aux Turcs). 
conserve le contrôle de Chônai, de Laodicée du Lykos et de la vallée du Méandre à l'issue des négociations conduites entre le sultan et Laskaris ${ }^{19}$.

Des quelques informations dont on dispose sur ces trois carrières, il ressort que les Maurozômai sont d'abord et avant tout des hommes d'armes et commandants militaires, qui, dès les années 1160 et jusqu'au début du $13^{\mathrm{e}}$ siècle, ont occupé à la cour impériale une position d'importance qu'ils ont su préserver. C'est vrai de Manuel comme de Théodore. Tandis que Nicétas Chôniatès omet de le présenter, hormis comme le beau-père du sultan, au contraire de Laskaris, Ibn Bībī le décrit comme «l'un des grands des empereurs de Byzance» (ou encore, suivant la double traduction proposée par Herbert W. Duda, «l'un des grands césars de Byzance») de même qu'Ibn alAthīr, qui ne le nomme pas, le désigne comme un «grand patrice ${ }^{20}$. Ce dernier ajoute qu'il accueillit le sultan dans sa citadelle de la région de Constantinople. L'origine péloponnésienne de la famille, suggérée par le double témoignage d'Eustathe de Thessalonique, est confirmée ultérieurement: en 1319 est mentionné dans une source latine un Maurosumi de Monemvasie $^{21}$. Il faut peut-être rapporter à cette implantation géographique la découverte au Pirée du sceau de Paul Maurozômès.

Aussi l'établissement de Manuel Maurozômès dans la vallée du Méandre continue-t-elle l'expansion amorcée par les Turcs dans la région dès les années $1170^{22}$, plus qu'elle ne sanctionne une stratégie proprement familiale ou locale. On hésite à suivre le témoignage de Nicétas Chôniatès qui fait de Manuel Maurozômès un prétendant au pouvoir impérial et un rival de Théodore Laskaris. Dans l'un de ses discours à Théodore Laskaris, il reproche à Manuel d'avoir cru pouvoir briguer le pouvoir pour lui-même et

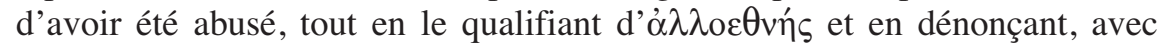

19. NicÉTAs ChôNIATÈs, Histoire, cité n. 8, p. 638 (Théodore Laskaris conclut la paix avec Kaykhusraw ${ }^{\text {er }}$, il assigne une part de son domaine au beau-père du sultan, Manuel Maurozômès, qui comprend la propre patrie de Nicétas Chôniatès, Chônai, la ville voisine de Laodicée de Phrygie et la vallée du Méandre) ; IDEM, Oratio 14 (CFHB 3), éd. J. L. Van Dieten, Berlin 1972, p. 136-137. Voir J. HofFMAnN, Rudimente von Territorialstaaten im byzantinischen Reich (1071-1210) (Miscellanea Byzantina Monacensia 17), Munich 1974, p. 61-63, 97-98; J.-C. Cheynet, Pouvoir et contestations à Byzance, cité n. 12, p. 469.

20. Iвn BīBĪ, Mukhtașar, cité n. 14, p. 30 ; IBN AL-ATHīr, al-Kamīl, cité n. 15, XII, p. 200. Les historiens ont habituellement retenu la traduction donnée dans le texte par Duda, «l'un des grands césars de Byzance», alors que Duda indique en note une alternative, «l'un des grands des empereurs (césars) de Byzance». Ibn al-Athīr (mort en 1233) utilise une expression très similaire. Sur l'emploi du terme «patrice» pour désigner les compagnons de l'empereur («le signe de la proximité impériale») chez les auteurs arabes, voir A. MiQuEL, La géographie humaine du monde musulman jusqu'au 11e siècle. II, Géographie arabe et représentation du monde : la terre et l'étranger (Civilisations et Sociétés 37), Paris 1975, p. 440-445.

21. A. E. Laiou-Thomadakis, The Byzantine Economy in the Mediterranean Trade System; Thirteenth-Fifteenh Centuries, DOP 34-35, 1980-1981, p. 205-206 ; H. Kalligas, Monemvasia, Seventh-Fifteenth Centuries, dans The Economic History of Byzantium. From the Seventh through the Fifteenth Century (Dumbarton Oaks Studies 39), dir. A. LAIou, II, Washington D.C. 2002 , p. 887.

22. P. WitTeK, Von der byzantinischen zur türkischen Toponymie, Byz. 10, 1935, p. 26-29;

C. CAHEN, La Turquie pré-ottomane (Varia Turcica 7), Istanbul-Paris 1988, p. 61-63. 
plus de virulence encore, sa trahison supposée ${ }^{23}$. Ibn Bībī, qui pourtant qualifie Maurozômès de «malik» à l'instar des fils du sultan Kilidj-Arslān (mais qui désigne l'empereur comme basileus), ne dit rien de telles prétentions au trône ${ }^{24}$. Quant à Georges Akropolitès, qui écrit plus de cinquante ans après les faits et dont la relation des actes du sultan s'accorde avec l'histoire d'Ibn Bībī (le baptême et l'adoption par l'empereur exceptés), il évoque les relations entre Alexis III, Théodore Laskaris et Kaykhusraw I ${ }^{\text {er }}$ sans jamais mentionner Manuel Maurozômès ${ }^{25}$. C'est encore l'image de transfuge dessinée par Nicétas Chôniatès qu'il faut nuancer: le ralliement de Manuel Maurozômès et de ses enfants au sultanat de Rūm au moment de la décomposition de l'empire est dans la continuité des relations privilégiées qui ont uni Alexis III et Kaykhusraw I ${ }^{\text {er }}$, avant et après 1203 (date à laquelle Alexis III quitte Constantinople), à Constantinople comme à Konya ${ }^{26}$.

C'est précisément dans le sultanat de Rūm et à son service que des Maurozômai sont connus au cours du $13^{\mathrm{e}}$ siècle. On rattache encore à cette famille un «émir Comnène », qui, suivant la chronique persane d'Ibn Bībī, fut actif dans les années 1220 sous le sultanat de Kayḳubād ${ }^{\mathrm{er}}$, fills et deuxième successeur de Kaykhusraw ${ }^{\mathrm{I}}{ }^{\text {r }}$. Homme de confiance de Kayḳubād I ${ }^{\mathrm{er}}$, il l'aida à mater plusieurs émirs, en récompense de quoi il fut élevé par le sultan à la fonction de beglerbeg ${ }^{27}$. Peu après, il se vit confier, avec un émir, le commandement d'une expédition militaire contre la Petite Arménie ${ }^{28}$. Nommé uniquement Comnène dans la version abrégée de l'œuvre (rédigée entre 1284 et 1285), il est aussi désigné comme Comnène Maurozômès (Mafrozom), à l'occasion de sa deuxième évocation, dans le manuscrit le plus ancien et le plus long de la chronique (un manuscrit de 1283, que Paul Wittek appelle «la copie de $1283 »$ ), ainsi que dans sa version turque (qui date du $15^{\mathrm{e}}$ siècle) ${ }^{29}$.

23. NicÉTAs ChôNiAtès, Oratio 14, cité n. 14, p. $136^{35}$.

24. IBN BīBĪ, Mukhtașar, cité n. 14, p. 30, 37.

25. Georges Akropolitès, Histoire, cité n. 16, 8-10, p. 14.

26. Ibidem. Pour R. MACRIDES, 1204: The Greek Sources, dans Urbs Capta. The Fourth Crusade and its Consequences (Réalités byzantines 10), dir. A. LAIOU, Paris 2005, p. 141-150, particulièrement p. 147-148, Alexis III continua à prétendre à l'exercice légitime du pouvoir. Ni Nicétas Chôniatès ni Ibn Bībī ne mentionnent, en revanche, l'implication d'Alexis III, aux côtés du sultan, dans la bataille d'Antioche-du-Méandre (en 1211, ou 1212 d'après F. VAN TrichT, La politique étrangère de l'empire de Constantinople, de 1210 à 1216. Sa position en Méditerranée orientale: problèmes de chronologie et d'interprétation [1 ${ }^{\mathrm{re}}$ partie], Le Moyen Âge 107, 2001, p. 223-227). Voir C. CAhEn, La Turquie pré-ottomane, cité n. 22, p. 68 ; GeORGES Akropolitès, The History (Oxford Studies in Byzantium), trad. com. R. MACRIDES, Oxford 2007, p. 37; D. KorobeiniKov, A sultan in Constantinople, cité n. 14.

27. Iвn BīBĪ, Mukhtașar, cité n. 14, p. 117-118, 120. Sur la fonction de beglerbeg, voir C. CAHen, La Turquie pré-ottomane, cité n. 22, p. 188-189: «On n'en trouve jamais nommé qu'un seul à la fois, et, dans les épisodes de la période mongole où nous est explicitement exposée la répartition des fonctions, le beglerbeg est toujours un personnage unique ».

28. IBN BīBĪ, Mukhtaṣar, cité n. 14, p. 131, 140-142.

29. Voir la présentation de l'œuvre, achevée au milieu de 1281, par H. W. DudA, s.v. Ibn Bībī, $E I^{2} 3$, 1975, p. 760-761. Je dépends ici des informations données par Wittek, puisque la traduction de Duda concerne la version persane abrégée. Duda signale néanmoins les variantes de la version longue, notamment la désignation à deux reprises de l'émir Comnène comme Comnène Maurozômès, ainsi que son identification avec le Comnène Maurozômès, beau-père du sultan Kaykhusraw I ${ }^{\text {er }}$ : voir İn BīBĪ, Mukhtaṣar, cité n. 14, p. 330 n. 83, p. 331 n. 86. 
Pour la première fois est mentionnée, incidemment, l'alliance entre les familles Comnène et Maurozômès, alliance confirmée par le sceau de Niğde et par une inscription des environs de Konya.

La famille des Maurozômai est en effet attestée ultérieurement, à la fin du $13^{\mathrm{e}}$ siècle, en territoire turc, alors que le sultanat seldjoukide de Rūm est passé sous la tutelle des Mongols. On a conservé jusqu'au $20^{\mathrm{e}}$ siècle, devant la porte d'une église située à environ 8 km de Konya ${ }^{30}$, une épitaphe grecque et chrétienne datée de 1297, rédigée en mémoire de Michel Amèraslanès ${ }^{31}$, peut-être un émir ${ }^{32}$. L'épitaphe, plusieurs fois commentée et justement célèbre, mentionne le grand-père de Michel, le $\pi \alpha v \varepsilon v \gamma \varepsilon v \varepsilon ́ \sigma \tau \alpha \tau o \varsigma$ Jean Comnène Maurozômès, puis son père, Jean Comnène, qualifié de $\tau \alpha \pi \varepsilon$ tvós (un moine donc). Le grand-père est lui-même qualifié d'arrière-petit-fils des empereurs porphyrogénètes. Homonyme, contemporain et revendiquant lui aussi son ascendance Comnène, le détenteur du sceau de Niğde, datable de la seconde moitié du $13^{\mathrm{e}}$ siècle, est identifiable avec le Jean Comnène Maurozômès cité dans l'épitaphe des environs de Konya.

L'inscription du sceau permet de préciser la généalogie de ce dernier sur un point: il est Maurozômès ou Comnène Maurozômès par son père. Franz Cumont, Paul Wittek et Kônstantinos Barzos ont reconstitué de manières diverses la généalogie du personnage, à partir de l'inscription, qui fait de ce dernier un descendant de Jean II et Manuel I ${ }^{\text {er }}$ (ou de son frère Alexis, seul autre empereur Comnène qui fût porphyrogénète), et de diverses sources narratives. Pas moins de trois, voire quatre hypothèses ont été émises pour déterminer l'époque et les protagonistes de l'alliance des Maurozômai avec les

30. La pierre est aujourd'hui au musée de Konya. Voir B.H. McLeAn, Greek and Latin Inscriptions in the Konya Archaeological Museum (Regional Epigraphic Catalogues of Asia Minor 4), Londres 2002, n 211.

31. L'inscription a été publiée en premier lieu par Cumont, sa lecture n'a pas été modifiée depuis en dépit des corrections proposées par Wittek. Voir F. Cumont, Note sur une inscription d'Iconium, BZ 4, 1895, p.99-105; P. Wiтtek, L'épitaphe d'un Comnène à Konia, Byz. 10, 1935, p. 505-515; IdEM, Encore l'épitaphe d'un Comnène à Konia, Byz. 12, 1937, p. 207-211; en dernier lieu (et sans aucune modification), B.H. McLeAn, Greek and Latin Inscriptions, cité n. 30. Sur le site de Sille, voir S. EyICE, Akmanastir (S. Chariton) in der Nähe von Konya und die Höhlenkirchen von Sille, $B F$ 2, 1967, p. 162-183.

32. Le terme n'a pas été élucidé et sa seule lecture continue de faire problème: amèr aslanès, amèras lanès ou amèraslanès. P. WITTEK, Encore l'épitaphe d'un Comnène à Konia, cité n. 31, p. 208-209, refuse d'y reconnaître un nom propre, qu'il s'agisse d'un nom de famille ou d'un nom personnel (pour Aslanès, soit Aslan ou Arslan), il en fait un sobriquet signifiant «lion de l'émir». Les arguments avancés me paraissent pour partie arbitraires. Ce peut être un titre. Je ne puis que mettre en relation ce terme avec un autre utilisé dans l'une des inscriptions de l'église

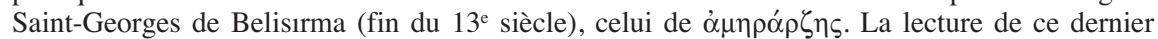
mot est elle-même aléatoire : sur les photographies on ne voit que AMHPA..H $\Sigma$. Voir N. THIERRY et M. Thierry, Nouvelles églises rupestres de Cappadoce. Région du Hasan Dă̆ p. 202-205 et pl.94; M. Restle, Die byzantinische Wandmalerei in Kleinasien, I, Reclinghausen 1967, p. 174-175, suivi par V. LAURENT, Note additionnelle. L'inscription de Saint-Georges de Bélisérama, REB 26, 1968, p.367-371, qui a proposé une nouvelle lecture, tandis que S. VRYONIS, Another Note on the Inscription of the Church of St. George of

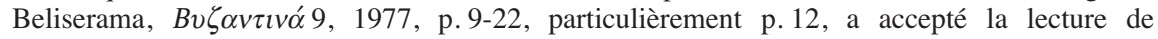
N. Thierry. 
Comnènes $^{33}$. En premier lieu, Cumont a identifié le Jean Comnène Maurozômès de l'inscription avec le prince de Trébizonde Jean Comnène Axouchos, qu'il suppose avoir épousé une Maurozômès ${ }^{34}$. Cette hypothèse, qui n'était étayée que par l'homonymie (partielle) des deux hommes, le contexte monastique et l'étroitesse des liens entre la principauté de Trébizonde et le sultanat et qui ignore le témoignage de la chronique d'Ibn Bībī, est contredite par la légende du sceau qui exclut que l'épouse de Jean soit une Maurozômès. En raison de sa fragilité, elle a été écartée, dès 1935 , par Wittek, qui fait valoir un élément important, la mention, pour les années 1220, dans la chronique seldjoukide d'Ibn Bībī, de l'émir appelé tantôt Comnène, tantôt Comnène Maurozômès. C'est ce dernier, «un serviteur fidèle du sultan», que Wittek identifie avec le Michel Comnène mentionné par Nicétas Chôniatès comme s'étant taillé un domaine indépendant dans la vallée du Méandre vers 1198 (en raison de la mention du nom de Michel et du titre d'émir dans l'épitaphe), qui, pour l'auteur, aurait épousé une femme de la famille des Maurozômai et aurait engendré Jean Comnène Maurozômès ${ }^{35}$. Encore une fois et pour la même raison que précédemment, la légende du sceau interdit cette hypothèse, puisque Jean ne peut être Maurozômès par sa mère. Wittek lui-même était conscient du caractère très hypothétique de cette reconstitution (concernant notamment l'ascendance et la carrière, entre le début du $13^{\mathrm{e}}$ siècle et les années 1220, de Michel Comnène). Aussi Barzos at-il raison de rejeter l'argumentation de Wittek (même s'il la déforme ou la comprend mal sur certains points) ${ }^{36}$. À l'inverse de Cumont et de Wittek, il s'intéresse moins aux Comnènes qu'aux Maurozômai, plus particulièrement à Manuel Maurozômès, beau-père du sultan de Konya. Jean Comnène Maurozômès aurait été le fils de Manuel Maurozômès, lui-même identifié, contre Wittek ${ }^{37}$, à l'émir Comnène Maurozômès ${ }^{38}$ et présenté comme le fils

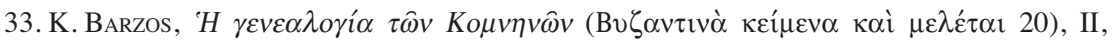
Thessalonique 1984, p. 498, rapporte, sans pour autant l'adopter, une hypothèse qui lui a été proposée par Szabolcs de Vajay, suivant laquelle Théodore Maurozômès aurait épousé une fille du porphyrogénète Alexis, frère de Manuel I ${ }^{\text {er }}$, et de Kataè de Géorgie.

34. F. Cumont, Note sur une inscription d'Iconium, cité n. 31.

35. P. W тттек, L'épitaphe d'un Comnène à Konia, cité n. 31, p. 509-514. Wittek considère que l'alliance entre les deux familles a eu lieu à cette génération en raison de la disparité de l'emploi de Maurozômès dans la chronique d'Ibn Bỉbī. L'émir serait un Comnène, qualifié occasionnellement et a posteriori de Maurozômès en raison de son alliance avec une Maurozômès.

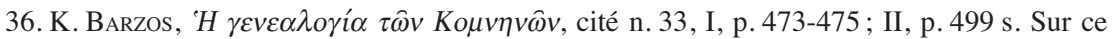
Michel, fils illégitime du sébastokratôr Jean Doukas, voir D.I. Polemis, The Doukai. A Contribution to Byzantine Prosopography (University of London Historical Studies 22), Londres $1968, n^{\circ} 45$, p. $91-92$.

37. P. WIтTEK, L'épitaphe d'un Comnène à Konia, cité n. 31, p. 510-511, refuse d'identifier Manuel Maurozômès et l'émir Comnène en dépit du texte de la chronique d'Ibn Bībī qui fait le rapprochement, considérant que la position de l'émir est incompatible avec celle de Manuel en 1205 (beau-père du sultan et «malik»). Voir à la n. 26 la réévaluation de la fonction de beglerbeg que sous-entend la définition donnée par Cahen. Wittek suppose que le passage de la chronique d'Ibn Bībī qui assimile l'émir Comnène à Manuel Maurozômès est une interpolation, étant absent de la version abrégée. Je ne puis me prononcer sur cette assertion, tout au plus 
de Théodore Maurozômès. C'est ce dernier qui aurait épousé une Comnène, en l'occurrence une fille illégitime de Manuel $\mathrm{I}^{\text {er }}$ Comnène ${ }^{39}$. Pour étayer cette hypothèse nouvelle, Barzos avance plusieurs arguments : le dévouement de Théodore envers l'empereur Manuel I ${ }^{\text {er }}$, le prénom de Manuel Maurozômès (identique, suivant cette généalogie, à celui de son grand-père Manuel I ${ }^{\mathrm{er}}$ ), les prétentions à l'empire de ce dernier, la conclusion par les sultans de mariages princiers. Si la fille de Manuel Maurozômès, qu'il épouse, est bien une arrière-petite-fille de Manuel $\mathrm{I}^{\mathrm{er}}$, le sultan s'allie avec les Comnènes ${ }^{40}$. On peut ajouter qu'Ibn Bībī (que Barzos ne semble connaître que par Wittek) signale bien l'existence d'un fils de Manuel Maurozômès, qui plus est au service du sultan ${ }^{41}$. Cohérente et en accord avec le témoignage d'Ibn Bībī comme avec la légende du sceau, bien qu'elle-même hypothétique, la reconstitution de Barzos de la généalogie des différents Maurozômai connus par les sources narratives et l'inscription peut être modifiée sur un point. Si Manuel Maurozômès, qu'Ibn Bībī qualifie de «malik» sans signaler son ascendance impériale, est bien le petit-fils de Manuel $\mathrm{I}^{\mathrm{er}}$, il est peut-être le fils du sébaste Jean Maurozômès, plutôt que de son contemporain Théodore Maurozômès, en raison du titre de Jean qui implique un lien de parenté avec la famille impériale ${ }^{42}$ et du remploi du même prénom deux générations plus tard, Jean Comnène Maurozômès portant, dans cette hypothèse, le prénom de son grand-père paternel ${ }^{43}$. Si Jean Comnène Maurozômès est mentionné dans l'inscription de 1297, on ne peut conclure, contrairement à Wittek, de l'absence de toute allusion à son décès qu'il est encore en vie à cette date; dans le cas contraire, on doit supposer une espérance de vie exceptionnelle ${ }^{44}$.

remarquer que Duda signale la variante de la version longue (la plus ancienne), sans la critiquer ni la mettre en cause. En l'état actuel des connaissances, on ne peut identifier cet émir Comnène Maurozômès qu'avec Manuel Maurozômès ou Jean Comnène Maurozômès (le détenteur du sceau).

38. Cahen suppose, avec plus de prudence néanmoins, une filiation similaire, dans Une famille byzantine au service des Seldjuqides d'Asie Mineure, Polychronion. Festschrift Franz Dölger zum 75. Geburtstag (Forschungen zur griechischen Diplomatik und Geschichte 1), éd. P. WIRTH, Heidelberg 1966, repris dans IDEM, Turcobyzantina et Oriens Christianus (Variorum Reprints CS 34), Londres 1974, VIII, p. 146: «C'est [Manuel Maurozome] encore, ou un de ses fils, qui, sous le nom plus ou moins valable d" "émir Comnène", aida Kaïqubâdh au début de son règne à dompter des officiers trop puissants et à conquérir des places-fortes arméno-ciliciennes, et reçut de lui, bien que toujours chrétien, le titre de beglerbeg. C'est apparemment un de ses descendants qui, jeune encore, mourut en 1297, ainsi qu'en fait foi dans une église proche de Qonya son épitaphe grecque conservée».

39. Voir l'analyse de la politique matrimoniale de Manuel I ${ }^{\mathrm{er}}$ par P. Magdalino, The Empire of Manuel I Komnenos, cité n. 10, p. 209-211, qui souligne l'exception que constitue alors 1'alliance conclue avec la famille des Maurozômai.

40. Sur «l'exportation» des filles Comnènes, voir R. MACRIDES, Dynastic marriages and political kinship, dans Byzantine Diplomacy, éd. J. ShePard et S. FrankLin, Aldershot 1992, repris dans EADEM, Kinship and Justice in Byzantium, 11th-15th Centuries (Variorum Reprints CS 642), Aldershot 1999, IV, p. 271-272.

41. IBn BīBĪ, Mukhtașar, cité n. 14, p. 38.

42. Voir J.-C. Cheynet, Pouvoir et contestations à Byzance, cité n. 12, p. 373.

43. Cette hypothèse m'a été suggérée par Jean-François Vannier.

44. Manuel Maurozômès a une fille mariée dès le début du $13^{\mathrm{e}}$ siècle. Son fils est né au plus tard à cette époque, il serait donc au minimum octogénaire en 1297. 
L'inscription du sceau précise sa généalogie paternelle, mais ne dit rien de son ascendance maternelle, peut-être d'origine turque. Les historiens ont noté les mariages mixtes conclus entre l'aristocratie grecque chrétienne et les Seldjoukides (et souligné, par exemple, la filiation grecque, par sa mère, du sultan 'Izz al-dīn).

L'ensemble de ces éléments, la découverte de l'épitaphe dans les environs de Konya, la conservation du sceau de Jean Comnène Maurozômès à Niğde, qui est au $13^{\mathrm{e}}$ siècle le siège de commandement d'une grande circonscription militaire du sultanat de Rūm ${ }^{45}$, confirme le fait que cette famille d'origine byzantine et chrétienne, à l'instar d'une branche de la famille mieux connue des Gabras ${ }^{46}$, s'est maintenue au moins jusqu'à la fin du $13^{e}$ siècle, en territoire turc et au service des sultans, alors même qu'elle continue d'être bien attestée dans l'empire, à cette même époque et dans les décennies suivantes ${ }^{47}$. Contemporain de Jean Comnène Maurozômès et qualifié comme lui de

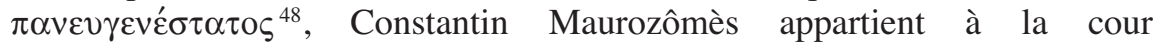
d'Andronic II Paléologue : mentionné, comme commanditaire, dans le colophon d'un manuscrit daté de 1286, il est connu pour avoir été ridiculisé et humilié, en lieu et place de sa maîtresse Stratègopoulina, par Constantin Paléologue, le frère d'Andronic II, à Constantinople en $1292^{49}$.

On ne s'étonne pas de l'usage d'un sceau de type byzantin dans le contexte turc. Dans le monastère même de Saint-Chariton, dans l'entrée de l'église, une inscription de 1288-1289 nomme le patriarche Grégoire [II], l'empereur des Romains Andronic [II Paléologue] et le grand sultan Mas $^{6}{ }^{4}{ }^{50}$. En Cappadoce précisément, quatre inscriptions peintes conservées dans des églises se réfèrent au règne de l'empereur byzantin: dans l'église de Karşı Kilise, à Gülşehir, l'inscription dédicatoire du 25 avril 1212, lacunaire, mentionne le règne de Théodore Laskaris ${ }^{51}$, comme l'inscription de l'église des Quarante Martyrs de Süveş, en 1216/1217, et celle de l'église principale

45. P. WiтTeK, s.v. Nīgde, $E I^{2}$ 8, 1995, p. 15.

46. Voir C. CAHEN, La Turquie pré-ottomane, cité n. 22.

47. D'autres Maurozômai sont attestés à la fin du $13^{\mathrm{e}}$ et au $14^{\mathrm{e}}$ siècle en Thrace (Ainos), en Asie Mineure occidentale (Smyrne), à Constantinople et à Lemnos. Voir PLP 7, $\mathrm{n}^{\text {os }} 17439$ 17444, p. 163-164.

48. Sur cette épithète, qui, dépourvue de valeur officielle, caractérise l'appartenance à la haute aristocratie (peut-être une ascendance liée aux Comnènes ou aux familles apparentées), voir D. KyrITSEs, The Byzantine Aristocracy in the Thirteenth and Early Fourteenth Centuries, Ph.D. diss., Ann Arbor 1997, p. 251-254.

49. A. Turyn, Dated Greek Manuscripts of the Thirteenth and Fourteenth Centuries in the Libraries of Great Britain (Dumbarton Oaks Studies 17), Washington D.C. 1980, p. 49-51; Georges Pachymérès, Relations historiques, éd. trad. A. FAILler, III (CFHB 24/3), Paris 1999, p. 175. Voir PLP 7, n 17443.

50. S. EYICE, Akmanastir (S. Chariton) in der Nähe von Konya und die Höhlenkirchen von Sille, $B F$ 2, 1967, p. 166-167.

51. C. Jolivet-Lévy, Images et espace cultuel à Byzance, dans Le sacré et son inscription dans l'espace à Byzance et en Occident. Études comparées (Byzantina Sorbonensia 18), éd. M. KaPLAN, Paris 2001, p. 167. Le sultan n'est pas mentionné, peut-être en raison du décès, en 1211 ou 1212 (voir n. 26), de Kaykhusraw I ${ }^{\text {er }}$ : voir EADEM, La Cappadoce. Mémoire de Byzance (Patrimoine de la Méditerranée), Paris 1997, p. 106. 
du monastère de l'Archangélos à Cemil, en 1217/1218 52 ; à la fin du $13^{\text {e }}$ siècle, dans l'église Saint-Georges de Belisırma (au sud-est d'Aksaray), ce sont le sultan Mas'ūd II et l'empereur Andronic II Paléologue qui sont cités par les dédicataires de l'église, la kyra Thamar et l'émir Basile Giagoupès. Catherine Jolivet-Lévy suppose que ce dernier, en raison de son prénom chrétien, de son patronyme porté par des Turcs comme par des Grecs, de son titre d'émir et de son habit (un long caftan et un turban blanc), est un vassal et un serviteur chrétien du sultan de Rūm ${ }^{53}$. Speros Vryonis mentionne la présence de Grecs à la cour, dans l'administration et l'armée seldjoukides, dont certains portent le titre d'émir ${ }^{54}$. Il note en particulier l'existence d'un bureau de notaires grecs dans la chancellerie du sultan. Suivant Claude Cahen, si le persan et l'arabe sont les langues du pouvoir et de l'administration seldjoukides, le grec est employé dans des lettres rédigées au nom de Kaykāūs ${ }^{55}$.

Ce ne sont pas seulement les pratiques administratives de l'empire qui sont en usage ou continuent de l'être dans le sultanat de Rūm, c'est aussi son idéologie. Jean Comnène Maurozômès et ses descendants soulignent, dans le sceau comme dans l'inscription, leur ascendance Comnène au point que le moine Jean semble avoir abandonné le nom de Maurozômès au profit du seul Comnène. C'est peut-être ce que reflète aussi la chronique d'Ibn Bībī en nommant l'émir de Kayḳubād Comnène plus fréquemment que Comnène Maurozômès. En rappelant et privilégiant, dans ce contexte proprement turc, leur ascendance Comnène, Jean Comnène Maurozômès et ses descendants agissent à l'instar des grandes familles aristocratiques contemporaines de la cour de Nicée et de celle des Paléologues, qui entendent ainsi légitimer leur rang ${ }^{56}$.

Sophie MÉTIVIER

Université Paris 1 Panthéon-Sorbonne

UMR 8167 Orient et Méditerranée

52. G. Kiourtzian, Une nouvelle inscription de Cappadoce du règne de Théodore ${ }^{\text {er }}$ Lascaris, DChAE 28, 2008, p. 131-137. Sur l'église et son décor, le peintre et le contexte d'élaboration de ce dernier, voir B. T. UyAR, L'église de l'Archangélos à Cemil: le décor de la nef sud et le renouveau de la peinture byzantine en Cappadoce au début du XIII ${ }^{\mathrm{e}}$ siècle, DChAE 28, 2008, p. 119-129.

53. C. Jolivet-Lévy, La Cappadoce. Mémoire de Byzance, cité n. 51, p. 113 ; N. ThIERRY et M. Thierry, Nouvelles églises rupestres de Cappadoce. Région du Hasan Dağı, cité n. 32, p. 202-207.

54. S. VRyonis, The Decline of Medieval Hellenism in Asia Minor and the Process of Islamization from the 11th through the 15th century (Center for Medieval and Renaissance Studies of the University of California), Berkeley 1971, p. 232-239, p. 233 (les notaires grecs).

55. C. CAHEN, La Turquie pré-ottomane, cité n. 22, p. 187.

56. Sur l'addition, comme marque de noblesse, du nom Comnène (ou Doukas) au $13^{\mathrm{e}}$ siècle, voir D. Kyritses, The Byzantine Aristocracy, cité n. 48, p. 220-221. 